\title{
Prevalencia y factores condicionantes de la obesidad en adolescentes de sexo femenino
}

\author{
Nutr. M. Angéljea Mardones H.1; Dr. Lduardo Atalah S.2
}

Etiological factors in female adolescent obesity

\begin{abstract}
A case control study was carried out to analyze the ctiological factors of obesity in 1147 female adolescents 15 to 18 ycars of age: 150 obese adolescents selected according to weight for height index $\geqslant 120 \%$ and total body fat $\geqslant 30 \%$ and 150 nomal adolescent matched by age. school level and socioeconomic status were studied. Dietary history, 3 day 3 physical activity record survey and weight for height index of parents were assessed in both groups. The mean energy intake was higher $(2478 \pm 607$ vs $2163 \pm 646$ keal $p<0.00 \mathrm{~J})$ and encrgy expenditure was lower $(1805 \pm 532$ vs $1998 \pm 737 \mathrm{kcal} \mathrm{p}<0.02)$ in cases than in controls, resulting in positive balances of 637 and 165 $\mathrm{kcal}$ respectively. An energy intake higher than those suggested by RDA and actual expenditure was observed in $62.2 \%$ of obeses and $41.6 \%$ of controls. A significantly higher prevalence of parental obcsity was found in obese adolescents with positive of negative energy balance in compatison with the control group (p<0.01). The data suggest that obcsity is the result of a complex relationship between energy balance and genctic factors which must be taken into account in preventive and therapeutic programs.
\end{abstract}

(Key wards: obesity, adolescent, energy intake, energy expenditure, parental obesity.

La obesidad representa el principal problema nutricional de los paises industrializados, donde alcanza alta prevalencia tanto en la adolescencia como en la vida adulta. Este fenómeno ha sido descrito también en los paises en vía de desarro1lo. donde coexiste con la desnutrición infantil. Desde hace más de 20 años, estudios nacionales han demostrado que la obesidad constituye un problema de Salud Pública en la población adulta, especialmente de sexo femenino, con una frecuencia creciente en los grupos de mayor edad. ${ }^{1}$ Estudios recientes confirman esta tendencia al demostrar $30,8 \%$ de obesidad en la mujer y $19,6 \%$ en el hombre, prevalencia que se correla-

1. Magister en Salud Púbtica, Instituto Profesional de Chillán.

2. Departamento de Nutrición, Facultad de Medicina, Universidad de Chile. ciona positivamente con la edad y negativamente con la talla y el nivel socioeconómico. ${ }^{2}$

En Chile la prevalencia de obesidad en los ninos menores de 10 afios es relativamente baja $y$ similar en ambos sexos. A partir de esa edad se produce un rápido incremento en el sexo femenino, llegando a afectar alrededor de un $25 \%$ de las adolescentes. ${ }^{3-5}$

La obesidad es un síndrome multicausal en cuya etiología participan numerosos factores entre los cuales destacan la ingesta energética, actividad física, factores genéticos, metabólicos, hormonales, etc. ${ }^{6-9} \mathrm{La}$ importancia relativa de cada uno de ellos va a depender de las características socioculturales de la población. El aumento excesivo de peso no sólo constituye un problema estético sino también un impacto negativo en la salud, por asociación con numerosas enfemedades y disminución de las expectativas de vida. ${ }^{10-12}$ Lew y col. demostraron un 
aumento importante del riesgo de muerte por diabetes, enfernedades cardiovasculares, digestivas, neoplásicas, etc., a medida que el índice de peso supera el rango normal. ${ }^{12}$

Existen numerosos enfoques terapéuticos para tratar la obesidad, pero todos ellos tienen un alto porcentaje de fracasos, ya sea por abandono del tratamiento o recaidas posteriores. Por lo tanto, lo lógico es encaminar los esfuerzos hacia la prevención primaria (impedir el desarrollo de la enfermedad) única foma efectiva de reducir la prevalencia. La prevención debe estar orientada con un criterio de riesgo, para lo cual es fundamental conocer la importancia relativa de los factores condicionantes, promoviendo su modificación en aquellos individuos más expuestos a desarrollar la enfermedad. El presente trabajo tiene como propósito conocer la prevalencia de obesidad y analizar los factores que la determinan en un grupo de adolescentes de sexo femenino que asisten a establecimientos de enseñanza media de Chillán.

\section{MATERIAL Y METODO}

[i] universo to constituycron 3.070 adolescentes do sexo femenino de ls a 18 años de edad, matriculadas en los establccimientos de enseñanza mcdia fiscales y partjeulares de la ciudad de Chiltán.

Pura determinas prevalencia de obesidad se utilizó una muestra probabilística con fijación proporcional estimada con $95 \%$ de confianza y $1 \%$ de precisión. Mediante un muestreo aleatorio simple sc seleccionaron 1.147 niñas, en las cuales se midió peso, talla y pliegues cutíncos (bicipital, tricipital, subescapular y suprail íaco) en condiciones estandarizadas.

I. diagnóstico del estado nutricional se baso en la relación peso-talla-edad según tablas de Jelliffe13 considerándose obesidad un indice de peso $>120 \%$ siempre que $3 e$ acompañara di una masa grasa $>30 \%$ del peso corporal, estimada según el criterio de Dursin. 14

En el total de casos de obesidad pesquisados ( $=153$ ) e igual número de controles de estado nutricional normal (peso-talla 90-110\%) pareados por edad. curso y nivel socioeconónico, se realizaton las siguientes determinaciones: encuestra de Graffar modificada para estimar nivel sociocconómico; ${ }^{15}$ encuesta alimentaria por tendencia de consumo cuantificada, para determinar consumo promedio diario de alimentos, a partir del eual y de la tabla de Composición Ouímica de los Alimentos Chilenos, se calculó la ingesta energetica, 16 la que se comparó con las recomendaciones del NRC, $1980 ; 13$ encuesta de registro de actividad física de tres días (encuesta de ticmpo-movimientoj a la cual se aplicaron los factores de gasto encrgético por actividad propuestos por Consolation, para estimar el gasto energético total diario; 17 peso y talla de los padres, con to cual se determinó el estado nutricjonal de los progenitores.

Durante el seguimiento se perdieron tres adolescentes en cada grupo y por to tanto en el análisis final se incluyen 150 casos (obesos) e igual número de controles (normales).

En el análisis estadístico se aplicaron pruebas de $t$ Student y $\mathrm{X}^{2}$, aceptándos como significativas diferencias con un $\mathrm{p}<0,05.1 \mathrm{~B}$
Tabla 1.

Distribución de la relación peso-taliaedad en 1.147 adolescentes de sexo femenino chillán

\begin{tabular}{lrr}
\hline $\begin{array}{c}\text { Peso-talla-edad } \\
\%\end{array}$ & $\mathrm{n}$ & \multicolumn{1}{c}{$\%$} \\
\hline$<90$ & 92 & 8,0 \\
$90-109$ & 710 & 62,0 \\
$110-119$ & 192 & 16,7 \\
$>120$ & 153 & 13,3 \\
\hline \hline Total & 1147 & 100,0
\end{tabular}

Fabla 2.

Pcso, talia, ingesta $y$ gasto energético promedio en adolescentes obesas y normales

\begin{tabular}{lrr}
\hline & Obesas & Normales \\
& $\bar{X} \pm$ D.E. & $\bar{X} \pm$ D.E. $\quad p$ \\
\hline Talla (cm) & $154,8 \pm 6,3$ & $155,5 \pm 5,2$ N.S. \\
Peso (kg) & $64,0 \pm 8,4$ & $49,9 \pm 4,4<0,001$ \\
Peso-talla \% & $130,1 \pm 11,3$ & $99,6 \pm 6,0<0,01$ \\
Ingesta energética & \\
$\quad$ Kcal & $2.478 \pm 607$ & $2.163 \pm 647<0,001$ \\
Gasto energético & \\
$\quad$ Kcal & $1.805 \pm 532$ & $1.998 \pm 737<0.01$
\end{tabular}

\section{RESULTADOS}

El estado nutricional de las adolescentes de acuerdo a la relación peso-talla-edad se presenta en la tabla 1, la mayor parte del grupo estudiado se encuentra en la categoría normal, aunque destaca un $30 \%$ con sobrepeso y obesidad $>110 \%$ ). Sólo un $8 \%$ de la muestra presenta un déficit en relación a este indicador.

En la tabla 2 se observan las características antropométricas, la ingesta y gasto energético promedio diario de los casos (obesas) y controles (nomales). En ambos grupos la talla es comparable pero existe una diferencia de $14,1 \mathrm{~kg}$. en el peso corporal $(p<0,001)$.

La ingesta energética recomendada según N.R.C. para este grupo de edad de $2.100 \mathrm{kcal}$. diarias, cifra que es claramente superada en el grupo de obesas y no asi en las nomales ( $p<0,001$ ). Para mantener el peso es necesario que el gasto energético sea equivalente a la ingesta. En el grupo de obesas el gasto es significativamente inferior al de los normales ( $p<0,01$ ) y bastante menor que la ingesta lo que determina un balance energético positivo de $637 \mathrm{kcal}$. diarias que equivalente a aproximadamente $70 \mathrm{~g}$ de tejido adiposo. Al calcular el gasto energético en relación al peso corporal se obtiene una cifra de $40,0 \mathrm{kcal}$. por $\mathrm{kg}$. de peso en las nommales y de $28,2 \mathrm{kcal}$ en las obesas acentuando las diferencias ya descritas. 
Es importante destacar la magnitud de las desviaciones estándares observadas tanto en la ingesta como en el gasto energético, lo que refleja que el grupo tiene un comportamiento muy variable. Por lo tanto es importante analizar no sólo el promedio sino también la adecuación de cada caso individual en relación a estas variables. información que se presenta en las tablas 3 y 4.

En cuanto a ingesta un porcentaje importante de las adolescentes estudiadas, consume más que la recomendación, proporción que es síghificativamente mayor en las obesas $(p<0,01)$. Destaca un grupo de obesas con una alimentación adecuada a sus necesidades e incluso un pequeño grupo con una ingesta insuficiente.

Otro aspecto que es importante analizar es la relación entre gasto energético e ingesta. Para mantener el peso constante el gasto energético debiera ser equivalente al consumo. to que se observa en una baja proporción de la muestra (tabla 4). En ambos grupos un alto porcentaje se encuentran en un balance positivo situación que es más frecuente en el grupo de las obesas ( $p<0,001)$. Llama la atención que $27 \%$ de las adolescentes nomales presentan balance negativo.

En la tabla 5 se analiza la frecuencia de obesidad en los padres, diagnosticada por un indice peso-talla $<120 \%$. Destaca que existe una significativa mayor frecuencia de obesidad familiar en el grupo de las obesas, ya sea de uno o de ambos progenitores $(\mathrm{p}<0.01)$. Este hecho podría sugenir un componente genético en la etiología de la obesidad, pero también puede reflejar hábitos alimentarios y patrones de actividad física distintos en los hogares de niños obesos y normales. Para tratar de separar ambos efectos se analiza la frecuencia de obesidad familiar en niños con balance energético positivo y negativo (tabla 6). En los obesos la presencia de obesidad familiar es mucho más alta lo que sugiere fuertemente la existencia de un factor
Tabla 3.

Adecuación de la ingesta energética en relación a las recomendaciones del N.R.C.

\begin{tabular}{lcc}
\hline $\begin{array}{l}\text { Adecuación } \\
\text { ingesta } \\
\%^{*}\end{array}$ & $\begin{array}{c}\text { Obesas } \\
\%\end{array}$ & $\begin{array}{c}\text { Normales } \\
\%\end{array}$ \\
\hline$<80$ & 5,4 & 15,4 \\
$80-119$ & 30,6 & 40,6 \\
$>120$ & 64,0 & 44,0 \\
\hline Total & 100,0 & 100,0
\end{tabular}

$\mathrm{X}^{2} 17,1 \mathrm{p}<0,01$

* \%de la recomendación del N.R.C.

Tabla 4.

Adecuación de la ingesta energética en relación a] gasto energético

\begin{tabular}{lcc}
\hline $\begin{array}{l}\text { Adecuación } \\
\text { ingesta } \\
\%^{*}\end{array}$ & $\begin{array}{c}\text { Obesas } \\
\%\end{array}$ & $\begin{array}{c}\text { Normales } \\
\%\end{array}$ \\
\hline$<80$ & 7,4 & 27,0 \\
$80-119$ & 25,2 & 34,3 \\
$>120$ & 67,4 & 38,7 \\
\hline \hline Total & 100,0 & 100,0
\end{tabular}

$\mathrm{X}^{2} 29,9 \mathrm{p}<0.001$.

* \% en telación al gasto energético.

Tabla 5.

Prevalencia de obusidad en los padres de Ins casos y controles

\begin{tabular}{lcc}
\hline $\begin{array}{l}\text { Obcsidad de } \\
\text { los padres }\end{array}$ & $\begin{array}{c}\text { Obesos } \\
\%\end{array}$ & $\begin{array}{c}\text { Normales } \\
\%\end{array}$ \\
\hline No & 19,2 & 63,1 \\
Uno & 48.2 & 30,4 \\
Ambos & 32,6 & 6,5 \\
\hline \hline Total & 100,0 & 100,0
\end{tabular}

$\mathrm{X}^{2} 59,9 \mathrm{p}<0,01$.

Tabla 6 .

Prevalencia de obesidad de los padres en relación al balance energético

\begin{tabular}{lcccc}
$\begin{array}{l}\text { Obesidad de } \\
\text { los padres }\end{array}$ & $\begin{array}{c}\text { Balance energético positivo } \\
\text { Obesas } \\
\%\end{array}$ & $\begin{array}{c}\text { Normales } \\
\%\end{array}$ & $\begin{array}{c}\text { Balance energético negativo } \\
\text { Obesas } \\
\%\end{array}$ & $\begin{array}{c}\text { Normales } \\
\%\end{array}$ \\
\hline No & 17,9 & 64,0 & 26,1 & 61,9 \\
Uno & 48,2 & 28,0 & 47,8 & 33,3 \\
Ambos & 33,9 & 8,0 & 26,1 & 4,8 \\
\hline \hline Total & 100,0 & 100,0 & 100,0 & 100,0 \\
& $\mathrm{x}^{2}=41,7 \mathrm{p}<0,01$ & $\mathrm{X}^{2}=12,5 \mathrm{p}<0,001$
\end{tabular}


genético. En otras palabras una alta ingesta energética promueve la obesidad en niños con antecedentes familiares de obesidad y no asi cuando la obesidad de los padres es menos marcada. lncluso una ingesta aparentemente baja (balance negativo) determina obesidad en adolescentes cuando los antecedentes familiares están presente en forma importante.

\section{DISCUSION}

En la mayor parte de los estudios nacionales la prevalencia de obesidad en mujeres adolescentes prácticamente duplica la cifra observada en el presente trabajo (13.3\%). Esta diferencia podria ser explicada por razones socioculturales que determinen características ambientales diferentes en Chillán en relación a la Región Metropotitana. donde se han realizado la mayor parte de los estudios. Sin embargo es probable que la principal diferencia está determinada por el estándar utilizado (peso-talla-edad) que implica una mayor exigencia en relación al estándar habitual. Tradicionalmente se ha aceptado que la relación peso-talla es independiente de la edad $y$ se ha aplicado un mismo nivel de normalidad durante toda la adolescencia, a pesar de que se producen importantes cambios de masa corporal durante el desarrollo puberal. El peso aceptable va a depender por lo tanto no sólo de la estatura sino también de la edad biológica definida según el criterio de Tanner. Así por ejemplo para una niña de $150 \mathrm{~cm}$ existen más de $5 \mathrm{~kg}$ de diferencias en el peso aceptable al comparar el periodo prepuberal con el estado 5 de desarrollo.

Idealmente debieran existir tablas de peso-talla en función del grado de desarrollo. Sin embargo como no hay estándares de referencia en este sentido, Jelliffe propuso tablas de pesotalla-edad como una aproximación parcial a este problema, criterio que utilizamos en el presente trabajo. El rango de peso norrial es claramente superior en el grupo de 15 a 18 años, en relación a los estándares tradicionales lo que determina una menor prevalencia de sobrepeso y obesidad. A pesar de este hecho, es importante destacar que $30 \%$ de la muestra presenta algún grado de sobrepeso, superando la magnitud de los problemas por déficit evaluados a través de este indicador.

La talla promedio del grupo reproduce lo descrito en mujeres adultas en los últimos 20 años, ubicándose por debajo de los estándares internacionales, sin que se observe una tendencia secular al crecimiento. Este retardo del crecimiento puede tener una base genética como lo ha planteado Valenzuela y Cols. ${ }^{19,20}$, aunque también podrja ser producto de una subalimenta- ción en periodo intermitentes del proceso de crecimiento y desarrollo.

La obesidad podría ser definida como un balance energético positivo en que el consumo supera las necesidades estimadas de acuerdo al gasto energético. La causa de la obesidad podría entonces ser definida analizando ambos factores, situación aparentemente fácil pero que presenta grandes dificultades metodológicas.

Todas las técnicas encuestales, (registro, recordatorio, pesada, observación) presentan limitaciones en su confiabilidad ya sea por problemas del encuestado o del encuestador. Por otra parte no existe necesariamente una buena correlación entre la ingesta reciente y el estado nutricional que refleja más bien la ingesta a través del tiempo. En el presente estudio se utilizó una encuesta de tendencia de consumo que representa mejor la ingesta habitual, aunque aparentemente su confiabilidad es menor. Debe tenerse presente $\sin$ embargo que el error encuestal es igual en ambos grupos. El grupo de obesas presentó un consumo claramente mayor al de las normales. superando en casi $400 \mathrm{Kcal}$ las recomendaciones promedio de energía para este grupo etáreo, lo que confirma que uno de los factores importantes en la etiología de la enfermedad es la alimentación hipercalórica.

$\mathrm{El}$ análisis del gasto energético implica probablemente mayores dificultades y las técnicas están menos estandarizadas para trabajo de comunidad. En el laboratorio las técnicas empleadas son la medición directa del consumo de $\mathrm{O}_{2} \mathrm{O}$ la estimación indirecta del consumo de $\mathrm{O}_{2}$ a partir del registro diario de la frecuencia cardía$\mathrm{ca}^{21-22}$ las que requieren de una infraestructura adecuada y no pueden aplicarse en grupos de población. La otra altemativa es registrar las actividades físicas realizadas por la persona durante todo el día (encuesta tiempo-movimiento) a to cual se aplican después factores promedio de gasto energético a cada uno de ellos, para estimar el gasto total diario. El método puede ser poco confiable en la medición exacta del tiempo y además por que el gasto puede ser muy variable para una misma actividad (caminar: lento, regular, rápido, etc.). Por otra parte el gasto energético registrado en un período breve de tiempo no refleja necesariamente la situación habitual. En este estudio el registro se realizó durante tres días consecutivos y, como en el caso de la ingesta, la magnitud del error se espera que sea similar en casos y controles.

Las obesas demostraron un gasto energético significativamente menor que las normales, diferencia que aumenta notoriamente al expresarla en $\mathrm{kg}$ de peso real. Se puede concluir por lo tanto que ta disminución de la actividad física repre- 
senta otro factor importante en la etiología de la obesidad.

Con todas las limitaciones analizadas, las obesas aparecen con balances energéticos francamente positivos $(+673 \mathrm{Kcal})$, que necesarianente van a traducirse en mayor depósito de tejido adiposo. Sorprende encontrar balance positivo en las normales ( $+165 \mathrm{Kca}$ ) lo que dentro de cierto grado puede considerarse normal ya que en esta edad se espera un aumento progresivo de la masa corporal hasta llegar al peso aceptable de un individuo. Lna exageración de esta tendencia podria significar tatnbién el desarrollo de sobrepeso ur obesidad. El bałance energético positivo se produec tanto por una mayor ingesta como por una menor actividad lísica. Sin embargo dada la magnitud de las desviaciones estándares es interesante evaluar el comportamento de cada caso cr particular.

La principal limitación de este cnfogue es tratar de establecer urla relacion entre ingesta en un periodo corto de tiempo y el estado nutricional que refleja una historia alimentaria bastantc más prolongada. A pesar de este hecho se observó que gran proporción de las obesas consumen excesos de calorias para sus necesidades estimadas por el peso aceptable. Sin embargo un tercio de este grupo presenta consumo adecuado e incluso inferior a sus necesidades. La interpretación de esta situación es dificil sobre todo al comprobar que una proporción importante de nomales también tiene un consumo superior a sus necesidades. Ello lheva a sugerir que e] factor genético podria ser importante ya que ur alto consumo determina obesidad sólo en un grupo de la población.

Una situación similar se observa al relacionar la ingesta con el gasto energético. Lo más frecuente es encontrar un balance positivo en el grupo de niñas obesas. Pero destaca un grupo significativo que tiene una ingesta adecuada al gasto energetico o incluso balance negativo. Dado el corto periodo de observación es difícil determinar si este hecho es habitual o circunstancia (régímen hipocalórico, enfermedad).

En las niñas nornales una proporción importante, aunque significativamente menor, mostró un balance positivo sin obesidad, to que nuevamente permite sugerir que existe un componente genético en la patogenia de la enfermedad. El análisis de la obesidad familiar parece confirmar esta hipótesis, ya que en las obesas con balance energético positivo y negativo se encuentra una proporción significativamente mayor de obesidad de los padres que en el grupo control.

Se podría concluir que la obesidad es una enfermedad multicausal donde interactían en proporciones variables una alta ingesta energé- tica, una baja actividad física y factores de tipo genético. Es importante por lo tanto lener presente estos factores para definir grupos de riesgo $y$ desarrollar acciones especificas tendientes a prevenir primariamente el desarrollo de la enfermedad.

\section{RESUMEN}

Con el propósito de evaluar los factores condicionantes de la obesidad se estudiaton 1.147 adolescentes de sexo femenino de 15 a 18 años. pertenecientes a 9 Liceos de Chillán. El diagrióstico de obesidad se basó en una relación pesotalla-edad $\geqslant 120 \%$ y en una masa grasa $\geqslant 30 \%$ del peso corporal. estimada según Durnin. Por el método de casos y controles se analizaron 150 obesas e igual numero de nomales. de nivel sociocconómico medio y bajo. Én cada uno de ellos se determinó ingesta energética por encuesta de tendencia de consumo, gasto energético a partir del registro de la actividad física durante 3 días y caracteristicas antropometricas de los padres. La ingesta energética de las obesas fue significativamente mayor respecto a las normales $(2.478 \pm 607$ vs $2.163 \pm 647 \mathrm{Kcal} p<0.001)$ y el gasto energético menor (1.805 \pm 552 vs $1.998 \pm$ $737 \mathrm{Kcal} \mathrm{p}<0,01)$ lo que determina un balance positivo de 637 y $165 \mathrm{Kcal}$ respectivajnente En $67,4 \%$ de los casos la ingesta era superior al gasto energético, proporción que disminuye a $38,7 \%$ en las normales $(p<0,001)$. Una significativa mayor prevalencia de obesidad familiar se observó tanto en obesas con balance energético positivo como negativo en comparación con los controles. Se concluye que la abesidad es el resultado de una compleja interacción entre ingesta, gasto energético y factores genéticos que deben tomarse en cuenta para la prevención y tratamiento.

\section{REFERENCLAS}

1. Valiente, S., Arteaga, A., Muñoz, M. at al: Obesidad un problema de salud pública. Rev Med Chil 91: $868,1963$.

2. Bove, M.J., Escobar, M.C., Taucher, E., Valiente, S.. Prevalence of over weight and obesity in 4.421 adults of different socioeconomic levels in Santiago. Chile. Abstracts XIII International Congress on Nutrition C 21: 159, Brighton, United Kingdom. 1985.

3. Atolah, E., Díaz, E., Araya, J., Arteagan A. et at. Evaluación Nutricional de una población infanto juvenil del . Area Norte de Santiago. Pediatría (Santiago) 22: 227, 1979.

4. Muzzo, S., Leiva, L., Zwoighaft, A.: Calidad de estatura y del estado nutritivo de escolares de la Región Metropolitana de Chile. Rey Chil Nutr 12: $185,1984$. 
5. Burrows, R., Díaz, L., Muzzo, S.: Estado nutritivo en adolescentes de clase media y baja. Rev Chil Nut 10: 129, 1982

6. Fisher, R., Fisher, D.A.: Current understanding of pathogenesis of obesity. South Med J 68: 931 , 1975.

7. Jomes, W.P.T. Thayhurh, P.: An integrated view of the metabolic arid genetic basis for obesity. Lancet 2: $770,1976$.

8. Straw, W.: The dilema of obesity. Current concepts of causes and management. Post Grad Med 72: 121 . 1982.

9. Uauy, $R$, Cariaga, L., Santand, $R$.: Obesidad infantil de causa nutricional. Rev Chí Nutr 12: 7, 1984

10. Van Itollie, $T \cdot B$.: Obesity: adverse effects on health and longevity. Am J Clin Nutr 32 (12 suppl): 2723 , 1979.

11. Stewart. A., Brook, R.: Effects of being overweight. Am J Public Health 73: 171, 1983.

12. Lew, E., Garfinkel. L.: Variations in mortality by weight among 750.000 men and women. J Chronic Dis 32: 563,1979 .

13. Suskind. R. Textbook of pediatric nuttition. Raven Press, New York. 290, 1981 .

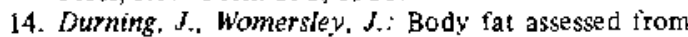
total body density and its estimation from skinfold thickness measures on 481 men and women aged from 16 to 72 years. Br J Nutr 32: ?7, 1974.
15. Graffar, M.: Une methode de classification sociale d'echantillons de population. Courrier 6: 455 , 1956.

16. Schmidt-Hebbel, H., Pennocchiotti, I. Masson, L. et al: Tabla de composición química de alimentos chilenos. 7a. ed. Santiago. Editorial Universitaria, 1985.

17. Consolazio, C.F., Matoush, L.R., Nelson, R.A., Torres, J.B., Isoac, G.J.: Environmental semperature and energy expenditures. I Appl Physiol 18: 65, 1963.

18. Snedecor, G., Cochran. W.: Statistical methods. 6a. ed. Anes, Iowa. Iowa State University Press, 548, 1972.

19. Volenzuela, C.: Adelanto de la pubertad de escolares chilenos respecto a escolares europeos medido por la velocidad de crecimiento estatural en estudio transversal. Pediatría (Santiago) 20: 74, 1977.

20. Vatenzuela. C., Avendoño, B.A.: Antropometría y maduración sexual de escolates de un área de Santiago de Chile. Bol of Sanit Panam 87: 74, 1979.

21. Spady, D.W.: Total daily energy expediture of healthy, tree ranging school children. Am J Clin Nutr 33: ?66, 1980.

22. Hreedson, P.S., Katch, V., Gilliam, T., Mc Connie, $S$. Energy expediture in prepubescent children: influence of sex and age. Am J Clin Nutr 34: 1827, 1981. 